\title{
Learning to Be a Good Tour-Guide Robot
}

\author{
J. Javier Rainer, Ramon Galán \\ Intelligent Control Group. Universidad Politécnica de Madrid \\ C/ José Gutiérrez de Abascal 2. 28006, Madrid, SPAIN \\ e-mail: javier.rainer@upm.es; ramon.galan(a) upm.es
}

\begin{abstract}
Thanks to the numerous attempts that are being made to develop autonomous robots, increasingly intelligent and cognitive skills are allowed This paper proposes an automatic presentation generator for a robot guide, which is considered one more cognitive skill. The presentations are made up of groups of paragraphs. The selection of the best paragraphs is based on a semantic understanding of the characteristics of the paragraphs, on the restrictions defined for the presentation and by the quality criteria appropriate for a public presentation. This work is part of the ROBONAUTA project of the Intelligent Control Research Group at the Universidad Politécnica de Madrid to create "awareness" in a robot guide. The softw are developed in the project has been verified on the tour-guide robot Urbano. The most important aspect of this propasal is that the design uses learning as the means to optimize the quality of the presentations. To achieve this goal, the system has to perform the optimized decision making, in different phases. The modeling of the quality index of the presentation is made using fuzzy logic and it represents the beliefs of the robot about what is good, bad, or indifferent about a presentation. This fuzzy system is used to select the most appropriate group of paragraphs for a presentation. The beliefs of the robot continue to evolving in order to coincide with the opinions of the public. It uses a genetic algorithm for the evolution of the rules. With this tool, the tour guide-robot shows the presentation, which satisfies the objectives and restrictions, and automatically it identifies the best paragraphs in order to find the most suitable set of contents for every public profile.
\end{abstract}

Keywords- Cognitive systems; learning; autonomous robot; fuzty systems; decision mating

\section{INTRODUCTION}

Antonomons robots are intelligent machines capable of performing tasks in the world by themselves, without explicit human control over their actions [1].

Within the development of multiple applications for a mobile robot, probably one of the first real world applications of indoor service robots has been mobile robots serving as tour guides in museums or exhibitions. We have developed our own interactive mobile robot called Urbano specially designed to be a tour guide in exhibitions [2]. The basic characteristics of Urbano are described in Section 3.

Our goal has been to create an automatic presentation generator that allows the flexible and dynamic display of information depending on the distinct kinds of audiences and other parameters that characterize the presentation. The antomatic selection of contents for composing sophisticated presentations is a non-trivial task. If the aims of the presentation, preference and interest for a particular snbject have to be taken into account, it becomes even more complex

The knowledge is based on an ontology of domainspecific concept words. Ontologies have been known in computer science as consensual models of domains of discourse, usually implemented as formal definitions of the relevant conceptual entities [3].

Some of the most recent works about automatic generation are: $[4,5,6,7,8]$. These works propose different architectures and methodologies than those presented here. What is original is the introdnction of fnzzy logic in the pruning of the resulting tree and in the quality index of the presentations.

This paper is focused on the automatic generation of presentations by a robotic system. Aspects concerning the grounding of the symbols used by the robot, e.g., paragraphs in the presentation or the learning of new concepts - have been circunvented for problems of space. In [9], the authors explore the problem of learning and the symbol-gronnding problem, and propose a systemic and integrative approach both problems.

\section{AUTOMATIC TEXT GENERATION.}

The history of natnral langnage processing is characterized by the influence of artificial intelligent $[10,11]$. A natural langnage generator generally has access to a wide knowledge field from which it must select infonmation to present to the users in various fonmats. Generating text is, therefore, a decision-making problem with mnltiple restrictions: knowledge, available linguistic tools, the objectives of the nser to whom the text will be directed, the situation, and past discourse. It deals with identifying the factors involved in this process and determining the factors that intervene in this process and its consequences $[11,12,13]$

An additional contribution of our work is to provide a dynamic framework that allows us to take into account each possible scenario. On the other hand, there can be no single general-purpose presentation format for all users, because each user differs in all aspects of interests and expertise levels, and in the devices used to visualize the requested information. These aspects are nsed as restrictions.

As a result, to prepare a presentation, the candidate items can be multiple, depending on knowledge server. The items 
selected in the generation process contain information about the theme of the presentation, but must as well include the items that are semantically related to the presentation. Thus, for example, a discussion of the painter Velazquez will also speak about the Italian painter Caravaggio due to his influence on Velazquez.

In our case a presentation generator is considered as a Cognitive Skill. It is assumed that skill development - e.g. giving presentations progressively more suitable to the public being addressed - is a fundamental architectural epiphenomenon. Rather than viewing a presentation as a mere form of communication, the focus here is on leveraging it as a means of expanding socio-communicative skills.

Several research projects have been undertaken to develop software tools for generating narratives, histories or presentations, and they have described many characteristics for generating presentations, but the quality criteria vary $[14,15]$.

The decision rules to establish the fact that a particular swap in the presentation strategy is useful and required are not clear yet. An interesting situation arises when the data changes and the environment are dynamic.

\section{A. Quality criteria of a presentation}

The quality of a presentation is defined by the different aspects that it characterizes, referred to in [16], as: nature, purpose, duration, and number of participants; for other authors, such aspects as connecting with the audience, the interests of the participants, the first and last minute, changes of rhythm, and "being natural and having fun" are very important. All the aspects have in common the difficulty of being effectively quantified for a computer program.

The prototype as developed has proposed the following quality criteria:

- Differences in time used and the anticipated time

- Time dedicated to the theme

- Time dedicated to entertainment

- Difficulty in understanding the theme

- Interest in the theme

- Time dedicated to related information (anecdotal)

- Time for interaction with the public

- Original information

- Non-conventional focus

These criteria are also used to obtain the assessment of the public.

\section{URBANO}

This Section describes the Urbano robot system, its hardware software and the experience we have obtained through its development and use until its actual mature stage.

\section{A. URBANO, an interactive Mobile Tour-Guide Robot}

This Section doesn't pretend to be an exhaustive technical description of algorithms, mathematical or implementation detail, but just an overview of the system.
Urbano robot is a B21r platform from iRobot, equipped with a four wheeled synchrodrive locomotion system, a SICK LMS200 laser scanner mounted horizontally in the top used for navigation and SLAM, and a mechatronic face and a robotic arm used to express emotions as happiness, sadness, surprise or anger.

The robot is also equipped with two sonar rings and one infrared ring, which allows detecting obstacle at different heights that can be used for obstacle avoidance and safety. The platform has also two onboard PCs and one touch screen.

The software is structured in several executable modules to allow a decoupled development by several teams of programmers, and they are connected via TCP/IP. Most of these executables are conceived as servers or services providers, as the face control, the arm control, the navigation systems voice synthesis and recognition, and the web server. The client-server paradigm is used, being the only client a central module that we call the Urbano Kernel. This kernel is the responsible of managing the whole system [17].

URBANO robot has a technology based on distributed application software. The recent version is an agent based on architecture that uses a specific CORBA approach as an integration tool. The robot has many functions: speaks, listens, navigates through the environment, moves his arm, responses to stimuli that affect its feelings.

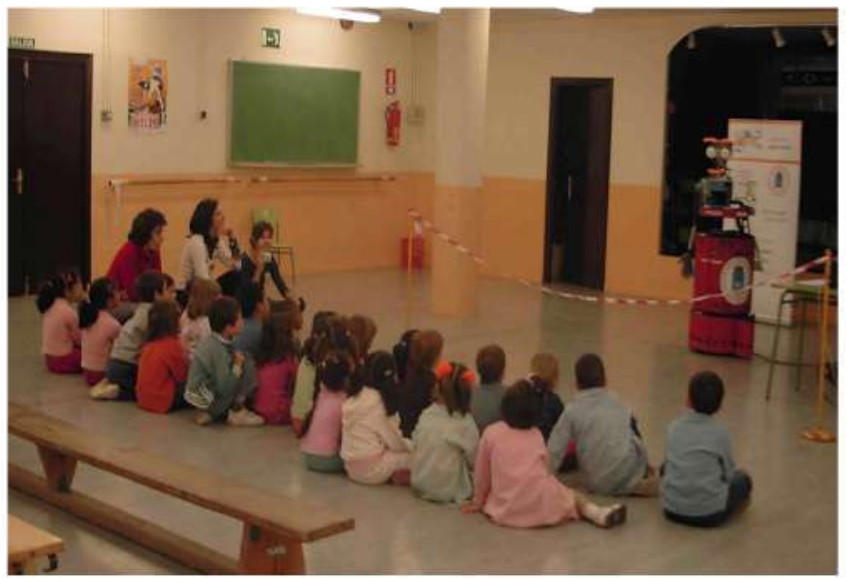

Figure 1. Urbano Tour-Guide Robot.

\section{B. URBANOntology}

The knowledge server consists of a Java application developed using the libraries of Protégé-OWL API. The tool is capable of reading and editing files in ".owl" format where the knowledge is stored in the form of ontologies and the management of the information from the kernel is made by means of messages that codify the request of specific information, and the reply is obtained from the server or the introduction of new data.

The functions of the knowledge server are: loading and saving ontologies; creating, renaming, and deleting classes or instances; displaying properties of a class; showing 
snbclasses or superclasses; showing or entering the value of a property; integrating one ontology into another; handling queries.

\section{APG AGENT}

$\mathrm{APG}$ agent software has been developed to be integrated in the architecture based on the agents that constitute the software of the Urbano robot. The developed computer application is activated on receiving a request from a user that selects a file that contains the pattern to be developed.

APG will request all the information from the knowledge server agent, nsing restrictions, that it needs to generate a file with the best presentation to be used by the system in the next performance by the robot.

Figure 2 shows this flowchart. It mnst be mentioned that presentations have been stored with quality indices (QI) for each of them, and in some cases, when faced with a reqnest very similar to a presentation already performed with high quality indices; this presentation will be used without needing to generate a new one.

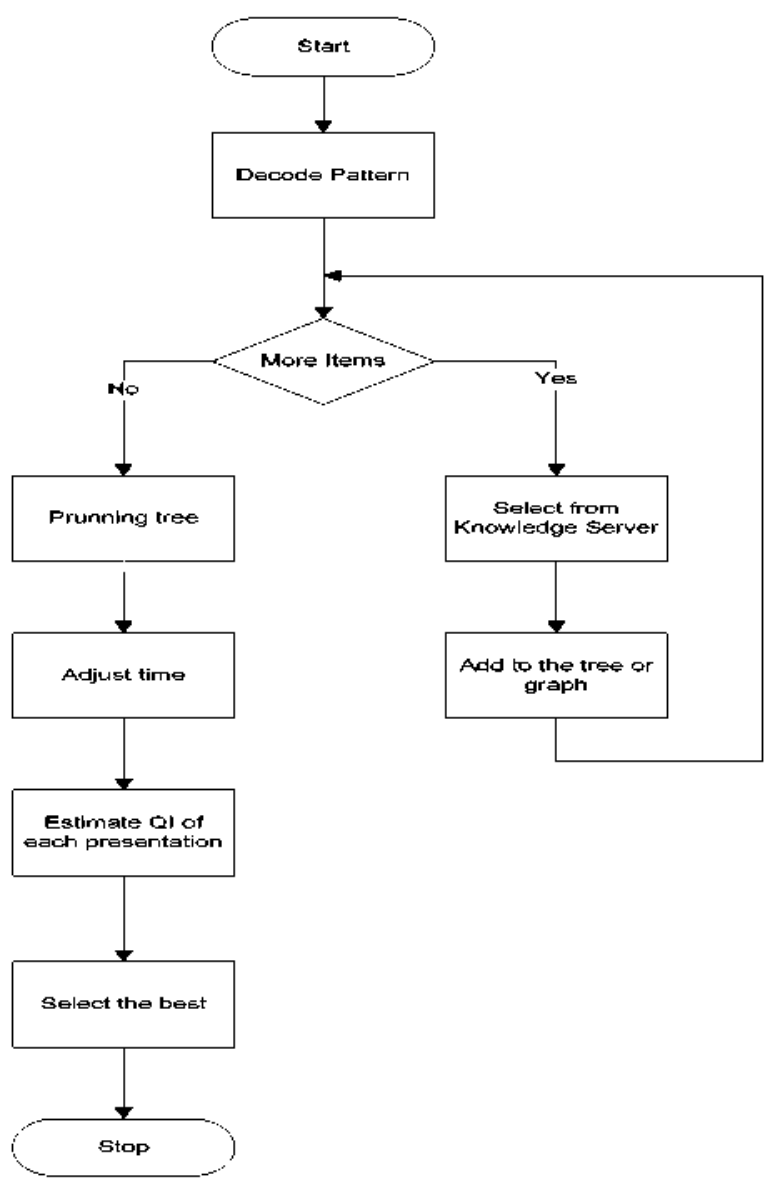

Figure 2. Flowchar APG

\section{PARAGRAPHS}

The paragraph is nsed by the robot as the minimum element of expression. It is assumed that the paragraphs have a size snch that coincidences or references between them lack meaning, and that they express a meaningful content. The following paragraph introdnces the painting "Las Meninas" by Diego Velazquez:

"A portwait of the infanta Margarita, daughter of Felipe IV (1605-1665), surrounded by her servants or 'family' in a hall of Madrid's Alcázar Palace.

This, the most famous of Velasquez's works, offers a complex composition built with admirable skill in the use of perspective, the depiction of light, and the representation of atmosphere.

There have been innmerable interpretations of this subject and later references to it. The most numerous emphasize a defense of the nobility of painting versus craft. Velasquez portrays himself, painting the painting itself, on the left of the canvas, thus affirming the supremacy of the art of painting. The infanta Margarita (1651-1673), wears white and appears in the center of the composition, surrounded by her ladies in waiting, the "meninas" Maria Agustina de Sarmiento and Isabel de Velasco, along with two court buffoons, Maria Bárbola and Nicolasito Pertusato, and a mastiff. Behind her; the duenna Harcela de Clloa convenses with the quartermaster, José Nieto, who is in the doonvav.

The King and Queen, Felipe $I$ and Maria de Austria (1634-1696) are reflected in the mirror at the back of the room, leading to series of extraordinarily complex spatial relations."

For each of the paragraphs there also exists a script that specifies the facial expressions and ann movements of the robot, the tone of voice and type of voice to nse, the position in the floor plan, and various details for interaction with the pnblic.

But in the ontology, each paragraph is "related" to the categories, which it belongs (e.g., in the previous paragraph: Is_a Painting, Is_a Description, Is_a Adult Level, Painted_by Diego Velazqnez, etc. These relationships are nsed for the selection of the paragraph).

\section{PATTERNS AND TREES}

The pattern, defined by the user, signals the elements that shonld form part of the presentation. For each item, these elements are established: its identification, its priority, its numerical order, its reference to the theme within available knowledge. There could be a limitation of time and a very large patter, in that case the priority index indicates the most important content to be included, but there could be the opposite case, that there is time left, then the system incorporates content that it is not initially considered.

It uses XML as the language to represent the patterns, which gnarantees an easy nse with different tools and programming languages. XML has emerged as a de facto standard for encoding and sharing data between varions applications. XML is also useful for stmctured information 
management, including information contained in knowledge server [18]. Our proposal is to design a software tool that helps the user to create and maintain the patterns.

$\mathrm{APG}$ requests from the knowledge server the paragraphs available for each theme, identified by its reference. The knowledge server will submit one or more paragraphs, becanse of a same concept can have several versions. Thns the pattern indicated in Figure 3 shows that first item of the presentation will be "mnsenm presentation" identified on the knowledge server as "Greetings" and it could involve, for example, three possible paragraphs, which will be included in the tree of possibilities, as is shown in Figure 4.

Some global restrictions including the level of audience will be used to prune the tree, eliminating the paragraphs that do not correspond to the reqnested level.

Three typical altemative heuristic searches have been tested to trim the tree. The first uses "brute force" to generate all the possible combinations and to group all the nnmeric values of the "quality criteria" of the paragraphs that forun the presentation, and then, using a set of fuzzy rules, estimates the quality index. It selects the presentation with the highest index. See Figure 5.

The second alternative uses "best-first search" so that as it goes along it takes the option that partially presents the best index. This altemative is without a doubt the fastest, but it cannot guarantee the selection of the best option.

The third altemative modifies the previous one so that it generates "backtracking" if the qnality index falls below a minimum.

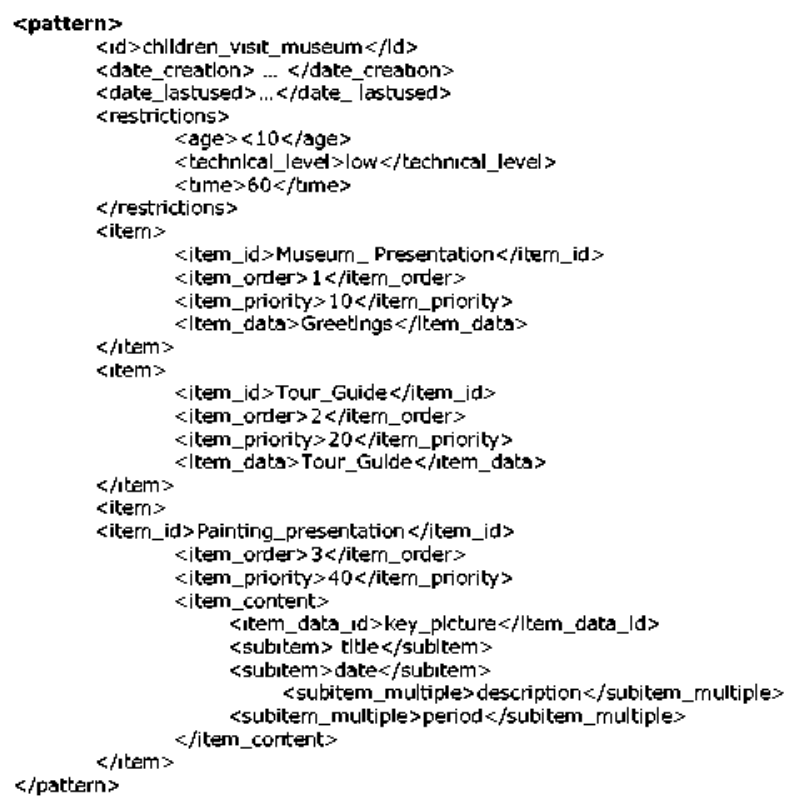

Figure 3. XML Patten Example

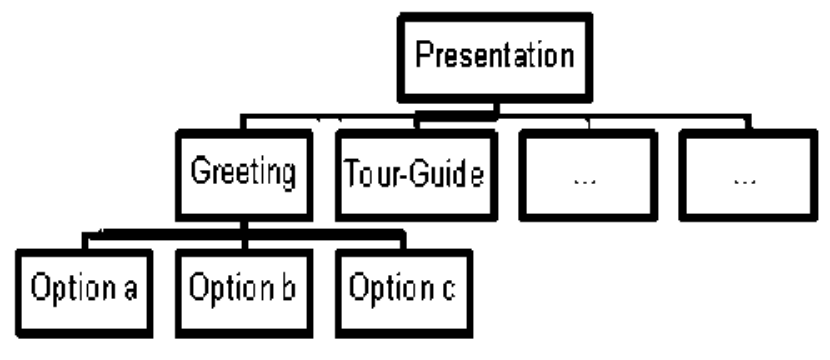

Figure 4. Tree Data Structure

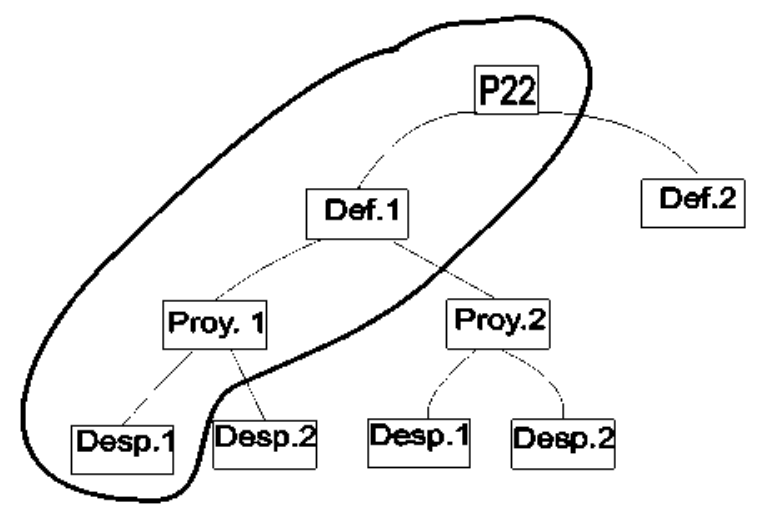

Figure 5. Different options

The presentation generated in this way analyzes the estimated time for its execution, and if this is greater than anticipated, it eliminates the paragraphs with the least necessary priority. On the other hand, if there is enough time, it includes some socially-oriented paragraphs such as jokes or comments abont sports, politics, or local events.

\section{DECISION-MAKING}

Decision-making is a part of the paradigm proposed by Zadeh [19] that has been currently examined in [20]. In a dynamic scenario as ours, and becanse of the natnre of the information that the system will handle, proper tools are needed to provide the intelligence for decision-making and supervision.

Decision-making is the cognitive process of selecting a course of action from multiple altematives. Fuzzy set approaches to decision-making are usually most appropriate when hnman evalnations and the modeling of hnman knowledge are needed [21].

The proposed solntion nses fuzzy rules to prune items to submit from the tree. It will use a variable that indicates the likelihood of inclnsion in the submission of a particular content. The fuzzy system will generate these values.

The fnzzy mles enable more flexibility. These rules will be adjusted and expanded.

All information available at the moment about the qnality criteria and its influence on the quality index is stored in the ontology of the knowledge server. 
The semantic network will indicate that the influence of time dedicated to the theme, expressed in a percentage, is VERY favorable if the time is VERY_HIGH bnt LOW favorable if the time is HIGH or NORMAL, and VERY nnfavorable in any other case. This relationship is defined by belonging to specified categories. In the developed prototype, the fnzzy rules nsed to calculate the quality index are obtained by consulting the categories that belong to a criteria in the knowledge server.

Five linguistic terms are defined: VERY_HIGH (VH), HIGH (H), NORMAL (N), LOW (L), VERY_LOW (VL). The fuzzyfication phase uses the function of membership to initially equidistant triangles, but in the leaming phase their centers can vary. The exit variable quality_index is also modeled with five terms and triangular funnctions. The techniqne of centroid method is nsed in the defuzzyfication phase.

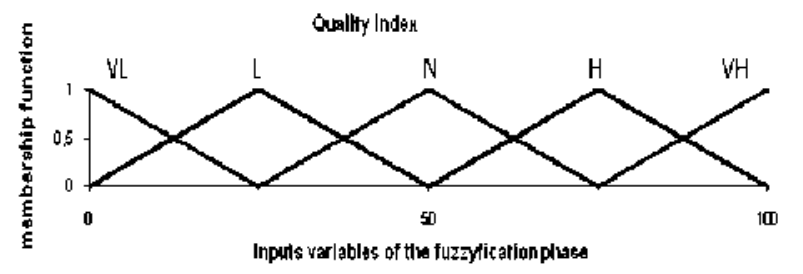

Figure 6. Inputs variables of the fuzzyfication phase

An interesting aspect is the partial quality estimation for the presentation. When considering three paragraphs (\#p 1,\#p2,\#p3), for example, it may happen that some criteria have unrepresentative valnes; if, for example, \#p $1, \# p 2, \# p 3$ correspond to paragraphs of technical description, the entertainment criteria will be null and the presentation will have a low estimation. In the tests made using pruning the best-first search, presentations are obtained wilh very poor estimations, and to compensate for this effect, an option of the best-first was introduced, bnt rejecting presentations with a low index.

If the categories of information expressed in the pattern are very generic, the nnmber of possible presentations increases enormonsly, bnt it permits the robot to generate higher quality presentations.

\section{LEARNING PHASE}

The most important characteristic of the proposal is the ability of the robot to learn. Initially it is thought that the robot will have a small number of quality criteria available to evaluate some presentations as good and others as bad, corresponding to the minimum level of education for a professional guide, in order to guarantee a minimum level of quality in its presentations.

A simple questionnaire has been designed that the public can fill out after attending a presentation by the robot. It asks for an evaluation of each quality criteria known at the time, indicating whether the robot should spend more or less time on each item, and a percentage evaluation of what the public considers valnable in the presentation. The Table I shows an example.

A proper statistical treatment of the qnestionnaires, eliminating the extremes and requiring a minimal quantity of data is perfonmed.

TABLE I. QUALITY CRITERIA

\begin{tabular}{|l|l|}
\hline Quality Criteria & $\begin{array}{l}\text { Should } \\
\text { be }\end{array}$ \\
\hline Time spent on the theme & - \\
\hline Time spent on entertainment & + \\
\hline$\ldots$ & \\
\hline New criteria to bear in mind & \\
\hline Answers to questions & 2 \\
\hline & \\
\hline Global evaluation & $(\mathbf{1 . . 5 )}$ \\
\hline & $\mathbf{4}$ \\
\hline
\end{tabular}

A genetic algorithm is nsed, an adjnstment of lingnistic terms and the membership functions, will penmit the quality index to be the closest to the average expressed by the public. To get a greater accuracy, the genetic algorithm is simultaneously used over several presentations as an attempt to offset the local minimals for a presentation.

The + and - indications are used to eliminate individual cases generated by the genetic algorithm in which, while still generating a correct quality index, the evaluation of the criteria mns contrary to public opinion.

In the tests carried out, it was shown that the "beliefs of the robot" about the qnality indices converge toward pnblic opinion, and as a result generate presentations that are evaluated more favorably.

\section{EXPERIMENTS}

A C++ based tool has been developed in order to implement both the fuzzy system and the genetic algorithms which are nsed for learning. Also, a standard MySQL database has been used for saving the presentations and the information that must be selected.

The prototype has let to validate the efficiency of the system to generate appropriate presentations to the imposed requirements. Various criteria for pruning the tree of possible presentations have been tried ont and it has been verified that the system is enongh fast for its real-time working.

In connection with the learning, various experiments have been done in order to validate the feasibility of the method and to have an estimation of necessary time. Simnlations have been performed with 5 linguistic terms and 4 variables or quality criteria; that means a total of 625 rules. It is nsed an archive file with 1300 presentations, whose quality is known for the opinion of the public. The genetic algorithm used has the following features: a genome composed by 625 variables, a population of 10 individuals, a crossing probability of 0.8 , and a mutation probability of 0.6 . 
This mntation probability, considerably higher than the usual 0.2 , has led to better results.

When there are no presentations covering all the possibilities in the archive file, some of the rules are never nsed, and the adjnstment of qnality index for these cases does not take place. Obviously if there are not experiences leaming does not take place.

As a future work, it is proposed to analyze the effect of introdncing, in real-time, new criteria which affect the quality index, and to analyze, if it is possible, the learning of features of quality for each available paragraph.

\section{CONCLUSION}

As a conclusion, special attention has been given to a mechanism for antomatic generation of presentations, analysis of search algorithms, learning phase and optimization of fnzzy logic rules, taking into account the intrinsic difficulty of natural language processing and antomatic generation.

The generator is a dynamic systen where knowledge will increase and, therefore, it will do the quality of the presentations. The robot will become increasingly better when making tour-guided visits. It also adds the ability to gesticnlate while is condncting the presentation: it specifies the facial expressions and ann movements of the robot, the tone and type of voice to use, the position in the floor plan, and several other details for interaction with the public.

This development will allow tour-guide robots to offer more affective leaming and a dynamic tour-guide visit, because the pnblic generally has become more sophisticated and, also, its expectations and demands. It provides a better nse of knowledge management. At the same time, advances in a new visitor-oriented approach, progress towards the creation of modnlar and scalable scenarios.

It presents a novel approach to the use of a computer ontology to represent the colpns that the robot works with, and the quality criteria for a presentation.

Methodology designed for the antomatic generation of presentations can be used for other applications related with decision making for autonomous robots. In the Urbano project design; there is a scheduler agent that selects the best task between different tasks, which the system has to do in a day. The criteria used for selecting the adequate task in short-term plan can be revised to optimize the long-term objective. A happiness model, as long-tem objective, and a decision-taking mechanism, as shor-term planner, was modeled for Urbano. Both use fuzzy logic and are adjusted by genetic algorithms that use the public opinion to leam to be a good tour-guide robot.

These systems have special importance to develop learning support for environments that require greater motivation and commitment, such as classrooms and workshops for stndents with special educational needs.

\section{ACKNOWLEDGMENT}

This work has been partially funded by Spanish Ministry of Science and Technology (ROBINT: DPI2004-07908-C0201 and ROBONAUTA: DPI-2007-66846-C02-01)

\section{REFERENCES}

[1] G. Bekey, Autonomous Robots: from biological inspiration to implementation and control, MIT Press books, 2005.

[2] D. Rodriglez-Losada, F. Matia, R. Galán, M. Hernando, J. M. Montero, and J. M. Lucas, Urbano, an Interactive Mobile Tour-Guide Robot. Advances in Service Robotics. Ed. H. Seok. In-Teh, 2008, pp 229-252.

[3] M. Uschold and M. Grüninger, "Ontologies: Principles, Methods, and Applicadions," Knowledge Eng. Rev, vol. 11, 1996, pp. 93-155.

[4] P. Barnaghi and S. Kareem, "Ontology- Based Multimedia Presentation Generation." IEEE TenCon, 2005.

[5] S. Bocconi. "Autonatic Presentation Generation for Scholarly Hypennedia," 1s International Workshop on Scholarly Hypertext at the fourteenth conference on Hypertext and Hypermedia (HyperText 2003), August 30. 2003.

[6] A Celentano and $O$. Gaggi, "Schena Modelling for Automatic Generation of Multitnedia Presentations." SEKE, 02 Lschia Italy, 2002.

[7] F. Frasincar and G. Hoube. "XML-Based Autonatic Web Presentation Generation," Proc, W'ebNet World Conference on W'ww and Internet, 2001.

[8] S. Little, J. Geurts and J. Hunter, "Dynatnic Generation of Intelligent Multimedia Presentations though Semartic Inferencing." The Sixth European Conference on Research and Advance Technology for Digital Libraries, Rome, Italy EDCL, 2002.

[9] J. Gómez, R. Sanz and R. Galán, "Leaming in Technical Systems: a Sign based Approach," In proceedings 1st International Workshop on Cognition for Tecluical Systems, Munich, 6- 7 October 2008.

[10] R. Cole, J. Mariani, H. Uszkoreit, A. Zaenen, and V. Zue, Survey of the State of the An in Hunan Language Technology. Canbridge: Canbridge University Press, 1996.

[11] K. Sparck Jones, Natural languages processing: a bistorical review. University of Cambridge, 2001.

[12] J. Allen, Natural Language Understanding. Redwood City, Ca.: Bernjamin Cummings, $2^{a}$ ed, 1995.

[13] J. Allen. "AI Growing Up. The Changes and Opportunities". AJ Magazioe, 19 (4), 1998.

[14] D. Jurafsky, and J. Martin, Speech and Language Processing. PTentice-Hall, 2000.

[15] K. Brooks, Metalinear Cinematic Narrative: Theory, Process and Tool. Phd Thesis, MIT, 1999.

[16] M. Cavazza, F. Charles, and S. J. Mead, "Character-based interactive storytelling." IEEE Intelligent Systems: Special Issue on AI in Interactive Enterlainment, 17(4):17-24, 2002.

[17] F. Laure, Técnicas de Presentación. Cecsa, 2002.

[18] M. Y. Maarouf and S. M. Chung "XML Integrated Environment for Service-Oriented Data Management," 20th IEEE International Conference on Tools with Artificial Intelligence. 2008.

[19] L. A Zadeh, "Fuzzy sets." Information and Control, 8. 338-353. 1965.

[20] L. A Zadeh. "Is there a need for fizzy logic?" Information Sciences. 178(13), 2751-2779, 2008.

[21] C. Kahraman. "Fuzzy set applications in industrial engineering", Infornation Science, Volume 177, Lssue 7, Pages 1531-15321, 2007. 